\title{
Klotho downregulation contributes to myocardial damage of cardiorenal syndrome in sepsis
}

\author{
FEI YAN ${ }^{1 *}$, YUE FENG $^{2 *}$, JIN CHEN $^{1}$ and JING YAN ${ }^{3}$ \\ Departments of ${ }^{1}$ General Practice, ${ }^{2}$ Radiology and ${ }^{3}$ Critical Care Medicine, \\ Zhejiang Hospital, Hangzhou, Zhejiang 310013, P.R. China
}

Received November 9, 2019; Accepted May 1, 2020

DOI: $10.3892 / \mathrm{mmr} .2020 .11178$

\begin{abstract}
Klotho is a type of single-pass transmembrane protein that is important for the proper function of numerous organs. The aim of the present study was to investigate the role of Klotho in sepsis-associated myocardial damage. In the present study, reverse transcription-quantitative PCR, western blotting and ELISA were conducted to examine the expression levels of function genes, and flow cytometry was performed to detect cell apoptosis and reactive oxygen species. The present study demonstrated that Klotho expression was significantly downregulated in septic mice and that the myocardial function of septic mice improved after treatment with exogenous Klotho protein. It further demonstrated that indoxyl sulfate inhibited the expression of Klotho protein. In addition, decreased Klotho protein further led to activation of the reactive oxygen species-p38 mitogen-activated protein kinase signaling pathway, finally resulting in myocardial damage. In conclusion, Klotho protein may be a key regulator in the myocardial damage of cardiorenal syndrome in sepsis. It also has a potential to be a therapeutic target for sepsis-associated myocardial damage in the future.
\end{abstract}

\section{Introduction}

It is well known that sepsis is a complex systemic disorder. Sepsis is defined as a dysregulated immunological host response to infection, usually caused by invading microorganisms and their products (1). As the most severe form of infection, sepsis can lead to multiple organ dysfunction

Correspondence to: Dr Jin Chen, Department of General Practice, Zhejiang Hospital, 12 Lingyin Road, Hangzhou, Zhejiang 310013, P.R. China

E-mail: chenjin_1110@163.com

Dr Jing Yan, Department of Critical Care Medicine, Zhejiang Hospital, 12 Lingyin Road, Hangzhou, Zhejiang 310013, P.R. China

E-mail: zjyyyanjing@163.com

${ }^{*}$ Contributed equally

Key words: Klotho, sepsis, myocardial damage syndrome and tissue damage $(2,3)$. It is estimated that $40-60 \%$ of cases of multi-organ dysfunction are associated with sepsis, including cardiorenal syndrome (CRS) (4). Sepsis-associated CRS is classified as type 5 CRS, which is characterized by a strong systemic inflammatory reaction that can result in simultaneous heart and kidney failure (5). Cardiac dysfunction during sepsis mainly presents as reduced cardiac contractility, impaired ventricular response to fluid therapy and progressive ventricular dilatation $(4,6)$. The mortality rate in patients with myocardial dysfunction may reach up to $70 \%$ (7). However, the molecular mechanisms of myocardial dysfunction in sepsis have yet to be fully elucidated.

Klotho is a type of single-pass transmembrane protein that is commonly expressed in renal tubes (8). The Klotho gene family includes three subtypes: $\alpha-, \beta$ - and $\gamma$-Klotho. Among them, $\alpha$-Klotho is the main type, the protective activity of which is crucial for the proper function of numerous organs (9). In addition, it has been confirmed that Klotho has antioxidative activity in acute kidney injury and cardiovascular disease $(9,10)$. In the present study, it was found that Klotho expression was decreased in mice with lipopolysaccharide (LPS)-induced sepsis. Klotho treatment could reverse the myocardial damage of CRS in sepsis. The data from the present study further demonstrated that indoxyl sulfate decreased the Klotho level and activated the reactive oxygen species (ROS)-mitogen-activated protein kinase signaling pathway in LPS-induced sepsis mice.

\section{Materials and methods}

Animal experiments. A total of 80 male wild-type C57BL/ 6 mice (aged 6-8 weeks, weight 20-24 g) were purchased from the Experimental Animal Center of Zhejiang Academy of Medical Sciences. All were housed at a specific pathogen-free laboratory (temperature, $20-24^{\circ} \mathrm{C}$; humidity, $50-70 \%$; free access to food and water; 12-h light/dark cycles). The animals were randomly divided into control and model groups [LPS group, LPS + Klotho group, LPS + activated charcoal (AST-120) group and indoxyl sulfate (IS) group]. There were 5 mice per group. The control group received no treatment. The LPS group was established through the intraperitoneal injection of LPS (Sigma-Aldrich; Merck KGaA) at doses of 5 , 10 and $20 \mathrm{mg} / \mathrm{kg}$. The LPS + AST-120 group, after LPS injection, received charcoal oral absorbent, 8\% AST-120 in powder 
diet for 3 weeks. The IS group was established through intraperitoneal injection for 4 weeks. The final concentrations of IS were 5, 10 and $20 \mu \mathrm{M}$, with the mice blood volume being $8.3 \%$ $\mathrm{x}$ weight. Pentobarbital sodium was administered through intraperitoneal injection at a dose of $50 \mathrm{mg} / \mathrm{kg}$ for anesthesia. The health and behavior of the mice were observed each day. The humane endpoints in the present study were followed to the greatest extent possible to avoid mice mortality, severe pain or suffering during the experiments. In addition, the mice were euthanized when exhibiting the follow symptoms: Weight loss of $15-20 \%$ of the original weight or no continuous weight gain during the growth period, displaying cachexia and persistent muscle wasting, loss of appetite [appetite loss for $24 \mathrm{~h}$ or poor appetite ( $<50 \%$ of normal amount) for 3 days] or weakness (inability to eat and drink, inability to stand for $24 \mathrm{~h}$ or extreme reluctance to stand). There was no accidental mortality in each group during the experiment. Finally, all the mice were sacrificed with euthanasia by cervical dislocation. Mice exhibiting no breathing or heartbeat were deemed to have succumbed in the present study. All animal experiments conformed to the guidelines established by the Ministry of Health (China) and were approved by the Animal Care and Welfare Committee of Zhejiang Hospital.

Hematoxylin and eosin $(H E)$ staining. Heart and kidney tissues were fixed with $4 \%$ formalin at room temperature for $24 \mathrm{~h}$, embedded in paraffin and sectioned into 4-mm thick sections. Subsequently, the tissue sections were deparaffinized in xylene and rehydrated in a series of graded alcohol solutions. Subsequently, the tissues sections were stained with hematoxylin-eosin (cat. no. C0105; Beyotime Institute of Biotechnology) according to the manufacturer's protocol. Stained sections were observed under a light microscope (magnifcation, $\mathrm{x} 200$ ).

Reverse transcription-quantitative $(R T-q)$ PCR. TRIzol ${ }^{\circledR}$ (Invitrogen; Thermo Fisher Scientific, Inc.) was used to extract the total RNA from kidney and cardiomyocyte tissue samples (50-100 mg) according to the manufacturer's instructions. Total RNA $(1 \mu \mathrm{g})$ was reversed into cDNA with a PrimeScript RT reagent kit (Takara Biotechnology Co., Ltd.). Prior to being reversed into cDNA, RNA samples were treated with DNase I recombinant and RNasin inhibitor. The primers used were: Atrial natriuretic peptide (ANP; sense: 5'-CGTATACAGTG CGGTGTCCA-3', antisense: 5'-GGTTGACTTCCCCAGTCC AG-3'), brain natriuretic peptide (BNP; sense: 5'-GCTGCT GGAGCTGATAAGAGAA-3', antisense: 5'-CGATCCGGTC TATCTTGTGCC-3') and GAPDH (sense: 5'-AGGAGCGAG ACCCCACTAACA-3', antisense: 5'-AGGGGGGCTAAG CAGTTGGT-3'). Each experiment was repeated three times. qPCR was performed using SYBR Premix Ex Taq (Takara Biotechnology Co., Ltd.). The PCR program used for the thermocycler (Bio-Rad Laboratories, Inc.) was $95^{\circ} \mathrm{C}$ for $5 \mathrm{~min}$, followed by 39 cycles at $95^{\circ} \mathrm{C}$ for $15 \mathrm{sec}, 58^{\circ} \mathrm{C}$ for $30 \mathrm{sec}$ and $72^{\circ} \mathrm{C}$ for $30 \mathrm{sec}$. The relative changes in gene expression were normalized to GAPDH and analyzed using the $2^{-\Delta \Delta \mathrm{Cq}}$ analysis method (11).

ELISA. Serum Klotho level was measured using a commercial ELISA kit (cat. no. AB15504; Sigma-Aldrich; Merck KGaA) based on the biotin double antibody sandwich technology. All serum samples were run in duplicate according to the manufacturer's protocol. The Klotho level was calculated as the average of duplicate samples.

Western blotting. For western blotting, protein samples were extracted from lysed tissues using RIPA (Beyotime Institute of Biotechnology) and the levels were detected with a bicinchoninic acid protein assay kit (Thermo Fisher Scientific, Inc.). Thereafter, protein samples with the same quality $(30 \mu \mathrm{g})$ were subjected to $10 \%$ sodium dodecyl sulfate-polyacrylamide gel electrophoresis and transferred to a nitrocellulose membrane (Merck KGaA) using a wet method. The membranes were blocked with $5 \%$ skim milk powder for $2 \mathrm{~h}$ at room temperature. Subsequently, the membranes were incubated at $4^{\circ} \mathrm{C}$ for overnight with primary antibodies targeted against: Klotho (1:1,000; cat. no. ab15504; Abcam), p38MAPK (1:1,000; cat. no. 8690; Cell Signaling Technology, Inc.), phosphorylated-p38MAPK (1:1000; cat. no. 5140; Cell Signaling Technology, Inc.) and GAPDH (1:1,000; cat. no. 5174; Cell Signaling Technology, Inc.). Subsequently, the membranes were incubated with a horseradish peroxidase-conjugated anti-rabbit IgG secondary antibody (1:100,000; cat. no. 70-GAR007; MultiSciences Biotech Co., Ltd.) for $1 \mathrm{~h}$ at room temperature. Protein bands were visualized using an enhanced chemiluminescence method (EMD Millipore) with the Odyssey imaging system (LI-COR Biosciences). Protein expression was quantified using Image Pro Plus software (version 6.0; Media Cybernetics, Inc.).

Flow cytometry for apoptosis and ROS detection. For cell apoptosis analysis, H9c2 cells (ATCC) were seeded $\left(2 \times 10^{5}\right.$ cells/well) and treated with IS $(5,10$ and $20 \mu \mathrm{M})$ for 24 and 48 h. Floating and attached cells were all harvested. Cell apoptosis was assessed using flow cytometry by labeling with the Annexin V-fluorescein isothiocyanate and propidium iodide apoptosis detection kit (MultiSciences Biotech Co., Ltd.). For ROS analysis, heart tissue and H9c2 cells were first incubated with dichlorodihydrofluorescein diacetate (H2DCFDA) at $37^{\circ} \mathrm{C}$ for $20 \mathrm{~min}$. The levels of intracellular $\mathrm{H}_{2} \mathrm{O}_{2}$ were measured using $\mathrm{H} 2$ DCFDA fluorescent dye (Abcam). Cell apoptosis and ROS levels were determined using a BD Accuri C6 flow cytometer (BD Biosciences) and analyzed using FlowJo software (version 10; FlowJo LLC).

Cell viability assay. Cell viability was examined using the Cell Counting Kit-8 (ccK8) assay (Beyotime institute of Biotechnology). Briefly, cells were trypsinized and seeded ( $2 \times 10^{3}$ cells/well) into 96-well plates. After culturing with IS for $48 \mathrm{~h}$ at $37^{\circ} \mathrm{C}, 10 \mu \mathrm{lCCK}-8$ reagent was added to each well and incubated at $37^{\circ} \mathrm{C}$ for $3 \mathrm{~h}$. Subsequently, the absorbance of each well was measured at a wavelength of $450 \mathrm{~nm}$ using a Multiskan MK3 spectrophotometer (Thermo Fisher Scientific, Inc.).

Equipment. The FACScan cytometer was purchased from BD Biosciences, the centrifuge was purchased from Eppendorf, the microscope was obtained from Olympus Corporation, the NanoDrop1000 spectrophotometer was purchased from Thermo Fisher Scientific, as was the cell incubator, and the 


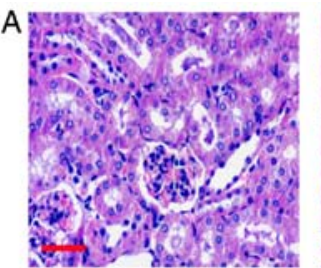

Ctrl

B

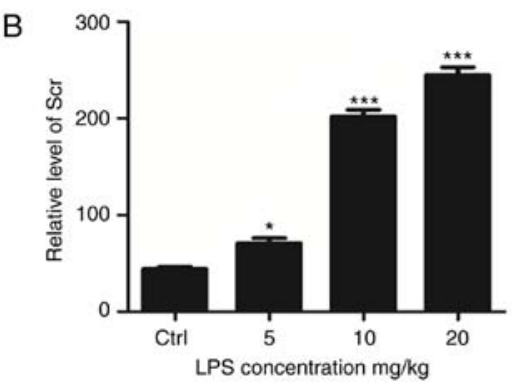

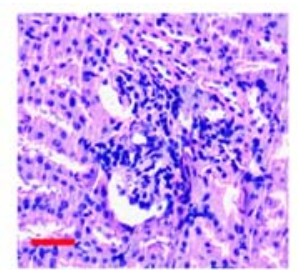

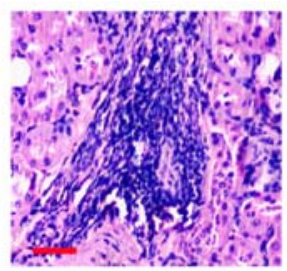

20

C

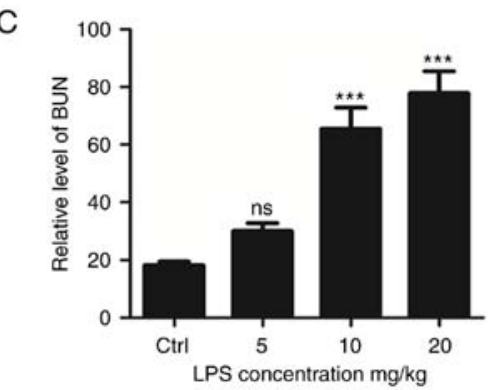

Figure 1. LPS injection induced kidney damage in mice. (A) Representative histopathological image of kidney tissue in the LPS-induced sepsis group at 24 h. (B and C) Levels of Scr and BUN in LPS-induced sepsis mice. Data are representative of 3 independent experiments ( $\mathrm{n}=5 \mathrm{mice}$ per group). Data are shown as mean \pm standard deviation. Scale bars $=100 \mu \mathrm{m}$. ${ }^{*} \mathrm{P}<0.01$ and ${ }^{* * * *} \mathrm{P}<0.001$. LPS, lipopolysaccharide; Scr, serum creatinine; BUN, blood urea nitrogen.

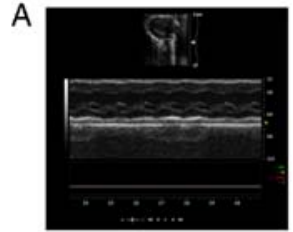

Ctrl

B

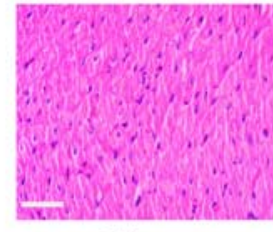

Ctrl

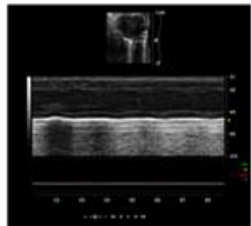

5

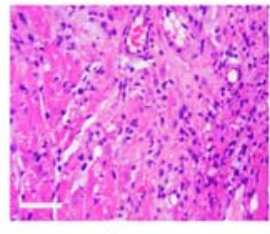

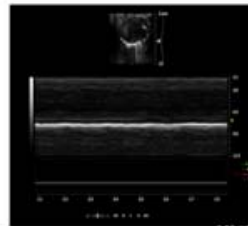

10

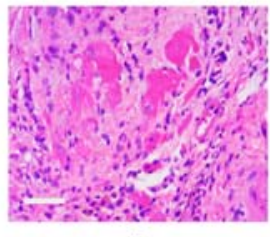

10

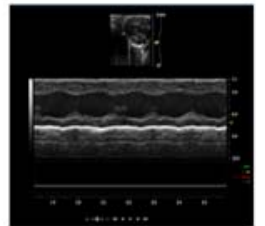

20

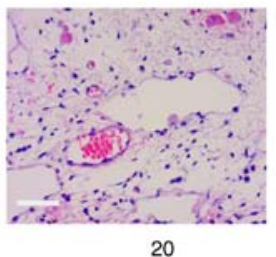

20
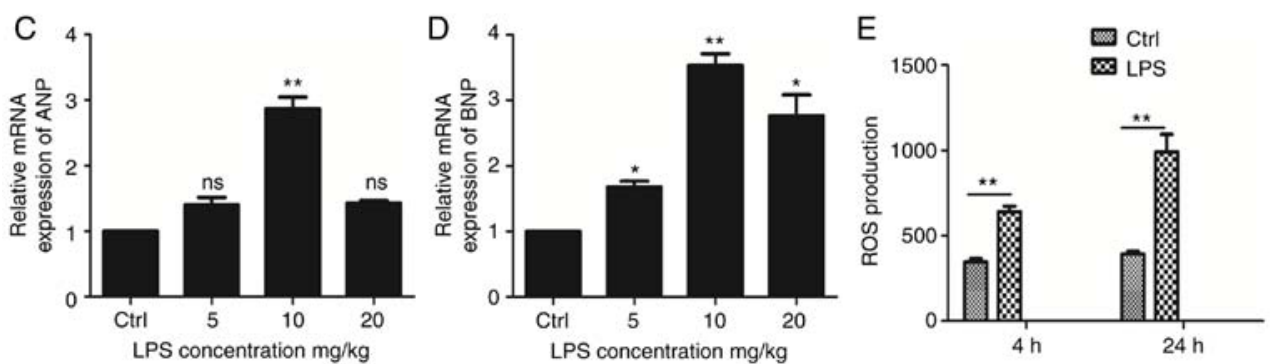

Figure 2. LPS injection induced cardiac dysfunction in mice. (A) Representative echocardiographic image of LPS-induced sepsis mice. (B) Histopathological changes of the myocardium in the LPS-induced sepsis group at $24 \mathrm{~h}$ shown by hematoxylin-eosin staining. Quantification of mRNA levels of (C) ANP and (D) BNP. (E) ROS production determined by ROS assay at 4 and $24 \mathrm{~h}$. Scale bars $=100 \mu \mathrm{m}$. ${ }^{*} \mathrm{P}<0.05$ and ${ }^{* *} \mathrm{P}<0.01$. LPS, lipopolysaccharide; ANP, atrial natriuretic peptide; BNP, brain natriuretic peptide; ROS, reactive oxygen species.

protein electrophoresis transfer device was purchased from Bio-Rad Laboratories, Inc.

Statistical analysis. All data are presented as mean \pm standard error of mean. For comparisons between two groups of normally distributed data, the Student's t-test was used. For multiple comparisons of normally distributed data, one-way analysis of variance was used followed by Tukey's test. $\mathrm{P}<0.05$ was considered to indicate a statistically significant difference.

\section{Results}

LPS-induced sepsis can lead to CRS in mice. CRS is a common complication of sepsis. To study the pathogenesis and pathological features of sepsis-related CRS, an LPS-induced sepsis mice model (LPS concentrations: 5, 10 and $20 \mathrm{mg} / \mathrm{kg}$ ) was established. The data from the present study demonstrated that LPS injection can successfully induce sepsis in mice. In addition, it was found that $10 \mathrm{mg} / \mathrm{kg}$ was the optimal LPS 

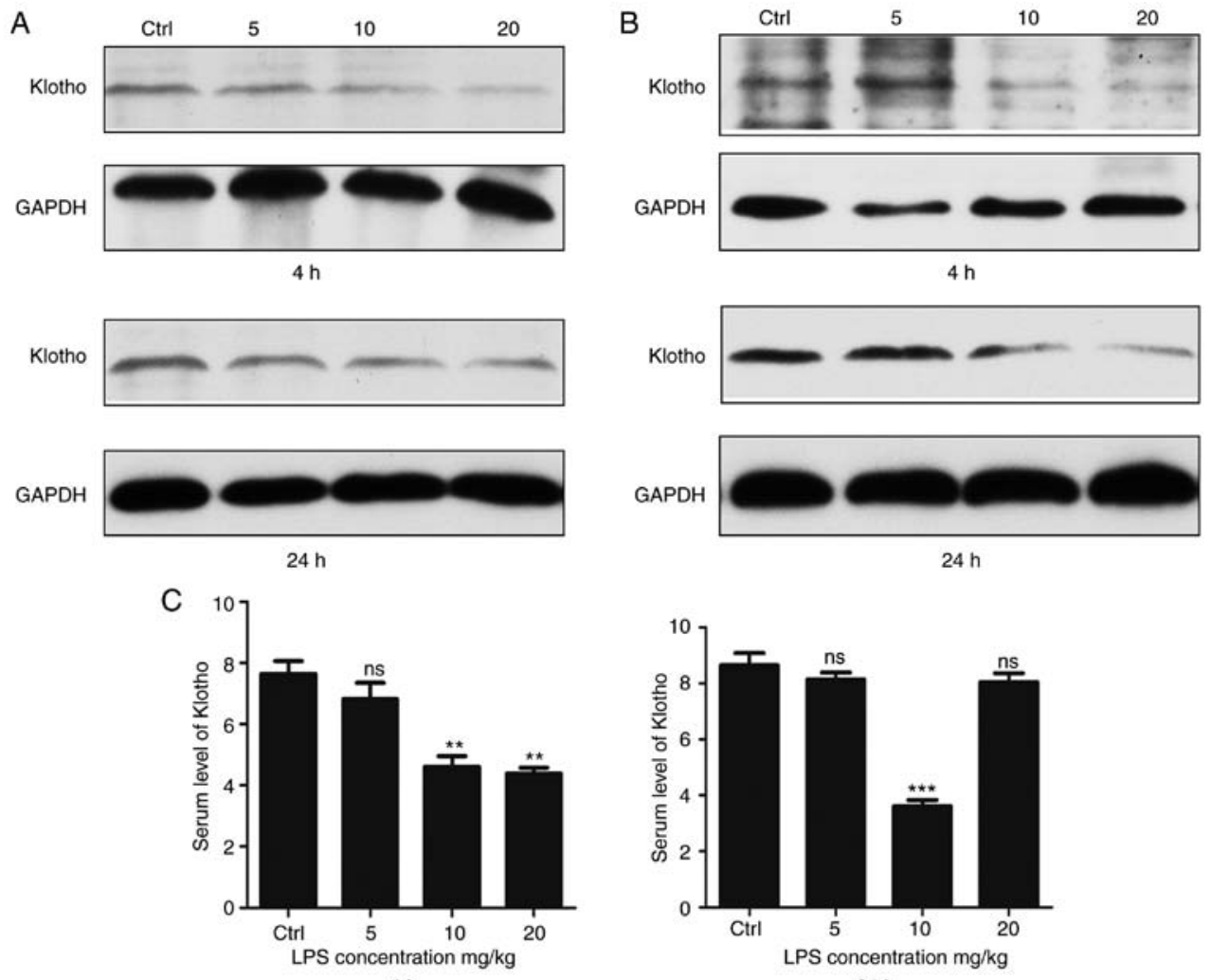

$4 \mathrm{~h}$

$24 \mathrm{~h}$

Figure 3. Klotho was decreased in LPS-induced sepsis mice. (A) Protein expression levels of Klotho in kidney tissue. (B) Protein expression levels of Klotho in myocardial tissue. (C) Serum levels of Klotho in LPS-induced sepsis mice. ${ }^{* *} \mathrm{P}<0.01$ and ${ }^{* * *} \mathrm{P}<0.001$. LPS, lipopolysaccharide.

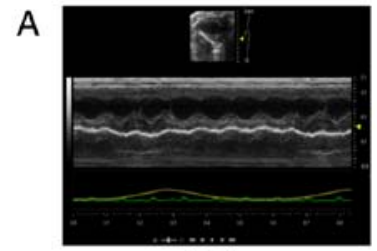

Ctrl

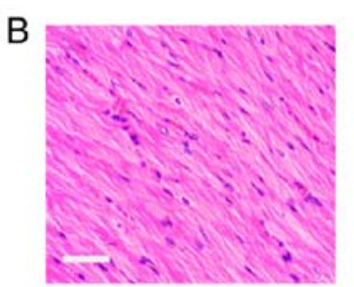

Ctrl

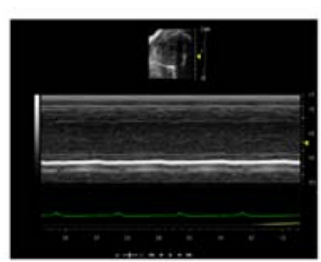

LPS

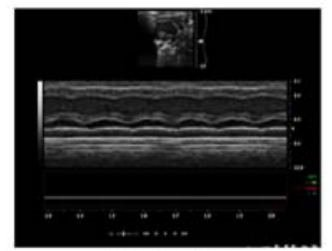

LPS+Klotho

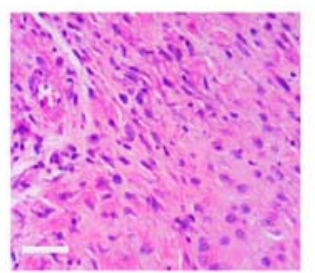

LPS
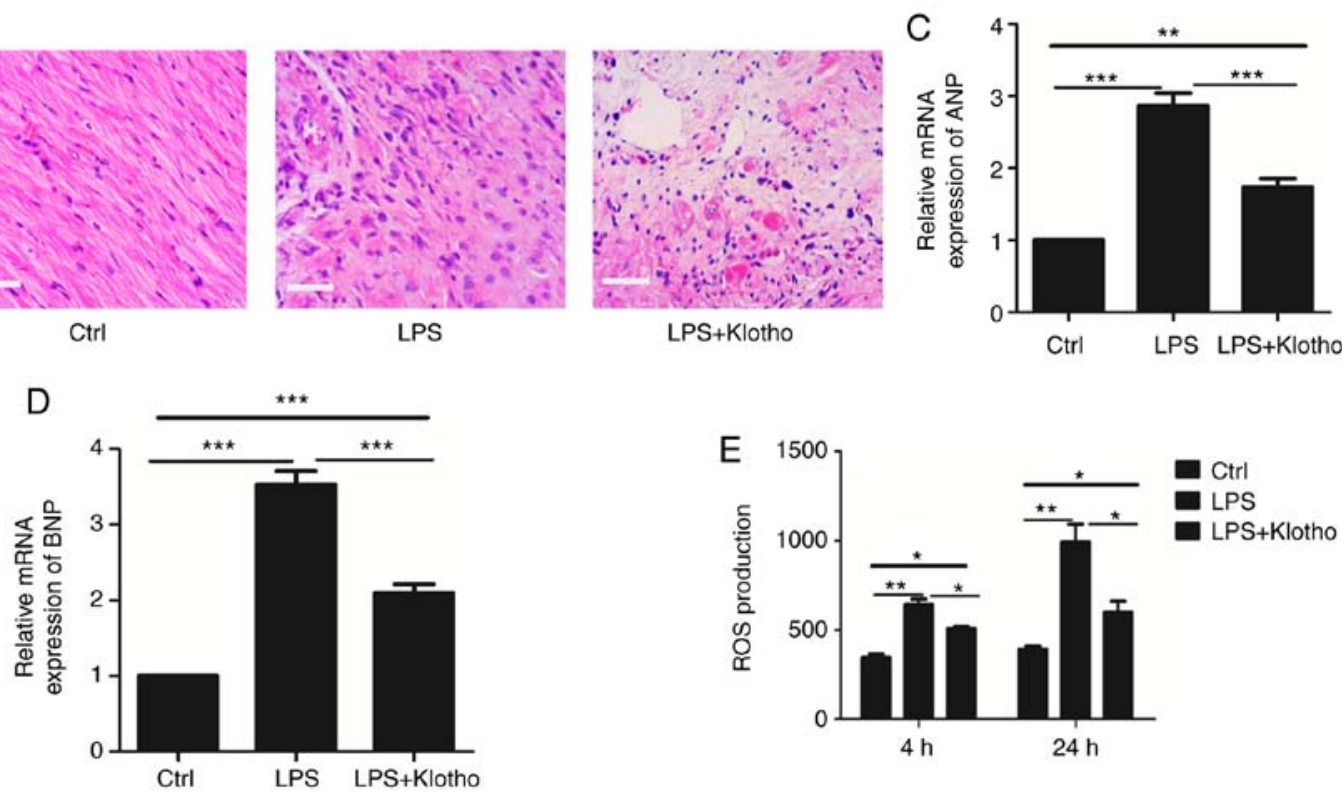

Figure 4. Klotho treatment prevented LPS-induced myocardial injury in septic mice. (A) Representative echocardiographic image of LPS-induced sepsis mice following Klotho treatment. (B) Hematoxylin and eosin staining of myocardial tissues following Klotho treatment. (C and D) mRNA levels of ANP and BNP measured using quantitative PCR. (E) ROS production in LPS-induced sepsis mice following Klotho treatment. Scale bars represent $100 \mu \mathrm{m}$. ${ }^{*}<0.05,{ }^{* * *} \mathrm{P}<0.01$ and ${ }^{* * *} \mathrm{P}<0.001$. LPS, lipopolysaccharide; ANP, atrial natriuretic peptide; BNP, brain natriuretic peptide; ROS, reactive oxygen species. 

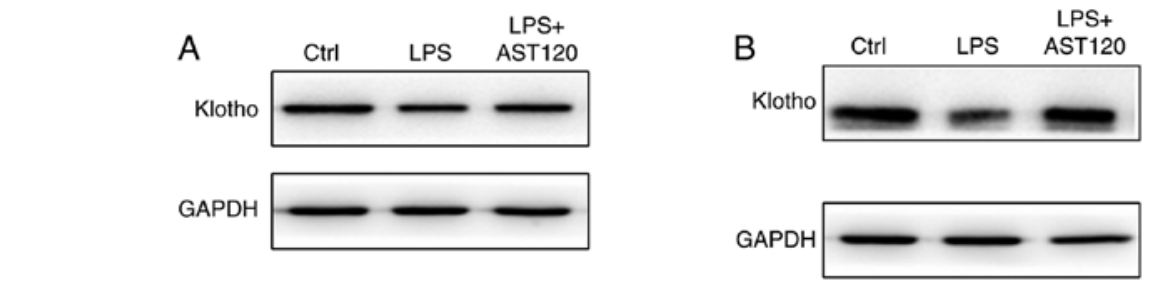
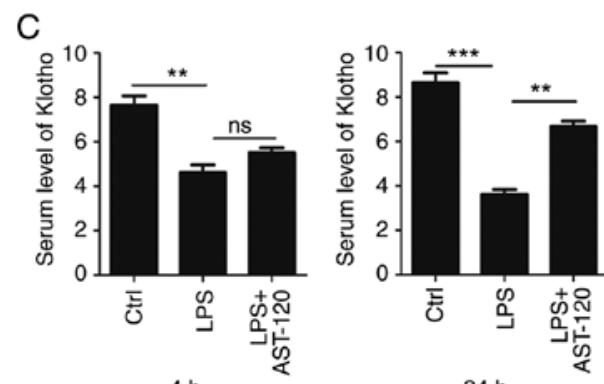

$24 \mathrm{~h}$

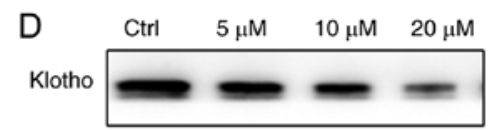

GAPDH

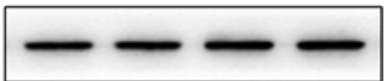

$\mathrm{F}$

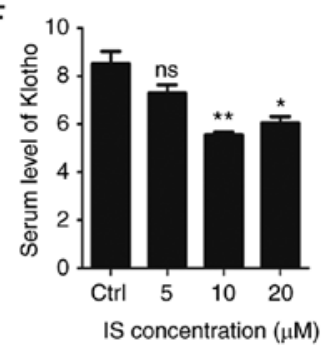

$4 \mathrm{~h}$

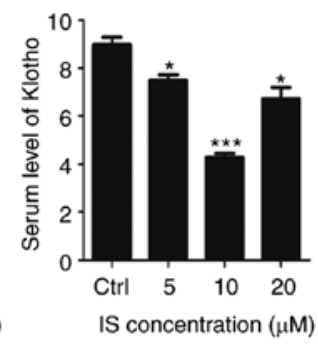

$24 \mathrm{~h}$

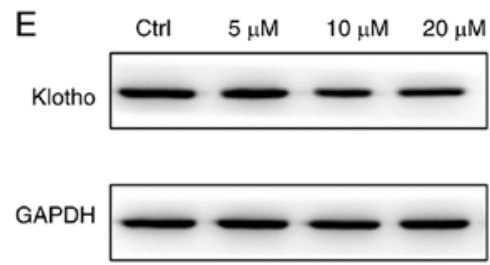

Figure 5. IS downregulated the expression of Klotho protein. (A) Protein expression levels of Klotho in kidney tissue after AST-120 treatment. (B) Protein expression levels of Klotho in heart tissue after AST-120 treatment. (C) Serum levels of Klotho in LPS-induced sepsis mice after AST-120 treatment. (D and E) Protein expression levels of Klotho in kidney and heart tissue after IS injection. (F) Serum levels of Klotho in IS-treated mice. ${ }^{*}<0.05$, ${ }^{* *} \mathrm{P}<0.01$ and ${ }^{* * * *} \mathrm{P}<0.001$. IS, indoxyl sulfate; AST-120, activated charcoal.

concentration. Thereafter, the heart and kidney functions of mice were evaluated. HE staining of renal tissue revealed obviously edematous glomerular and tubular epithelium cells, as well as a large number of inflammatory cell infiltrates in LPS-induced sepsis mice. By contrast, the structure of the glomerulus and tubules was clear and normal in control mice (Fig. 1A). The levels of serum creatinine and urea nitrogen were significantly higher in LPS-induced sepsis mice (Fig. 1B and C).

Cardiac dysfunction is another clinical feature of sepsis. Cardiac echocardiography revealed that LPS-induced sepsis mice had left ventricular systolic dysfunction and decreased cardiac contractility (Fig. 2A). Evaluation of the myocardial tissue morphology demonstrated that the myocardial gap of the LPS-induced sepsis group was broader than that of the control group, cardiac cells had severe edema, myocardial hyperemia was evident, adherent inflammatory cells were increased and some myocardial tissues demonstrated myocardial degeneration and dissolution (Fig. 2B). The expression of myocardial injury markers such as ANP and BNP were detected and, as shown in Fig. 2C and D, the expression of ANP and BNP was upregulated in the LPS-induced sepsis group. In addition, the ROS production of cardiomyocytes was also increased in LPS-induced sepsis mice (Fig. 2E).

Klotho protein is significantly decreased in LPS-induced sepsis mice. It has been reported that Klotho protein plays an important role in numerous organs, such as the kidney and heart $(12,9)$. To ascertain whether Klotho protein can affect the development of LPS-induced sepsis, the level of Klotho was detected in LPS-induced sepsis mice. As shown in Fig. 3A, the Klotho protein level of kidney tissue was significantly decreased in the LPS-induced sepsis group. The data further revealed that the expression of Klotho protein was also downregulated in myocardial tissues and that the downregulation of Klotho protein was dependent on the LPS concentration (Fig. 3B). Serum samples were collected for ELISA, which indicated that the level of Klotho protein was decreased compared with that in the control group (Fig. 3C).

Klotho protein treatment reverses myocardial injury in $L P S$-induced sepsis mice. To further investigate the function of Klotho in the myocardial damage of CRS in sepsis, a Klotho protein intervention experiment was next performed. After LPS injection at a dose of $10 \mathrm{mg} / \mathrm{kg}$ for $24 \mathrm{~h}$, the mice were treated with Klotho protein $(0.02 \mathrm{mg} / \mathrm{kg})$ through intraperitoneal injection for 4 days. The results indicated that the myocardial function of LPS-induced sepsis mice was improved after Klotho protein injection (Fig. 4A and B). Similarly, the expression levels of ANP and BNP were downregulated compared with the LPS-induced sepsis group (Fig. 4C and D). In addition, the ROS production of cardiomyocytes was also decreased compared with that in the LPS-induced sepsis group (Fig. 4E). These results confirmed that Klotho had an important role in the myocardial damage of CRS in sepsis.

Klotho downregulation is associated with redundant IS. As Klotho is essential in CRS, the cause of Klotho downregulation required investigation. It is known that uremic toxins often accumulate in sepsis. IS is a well-known protein-bound 
A
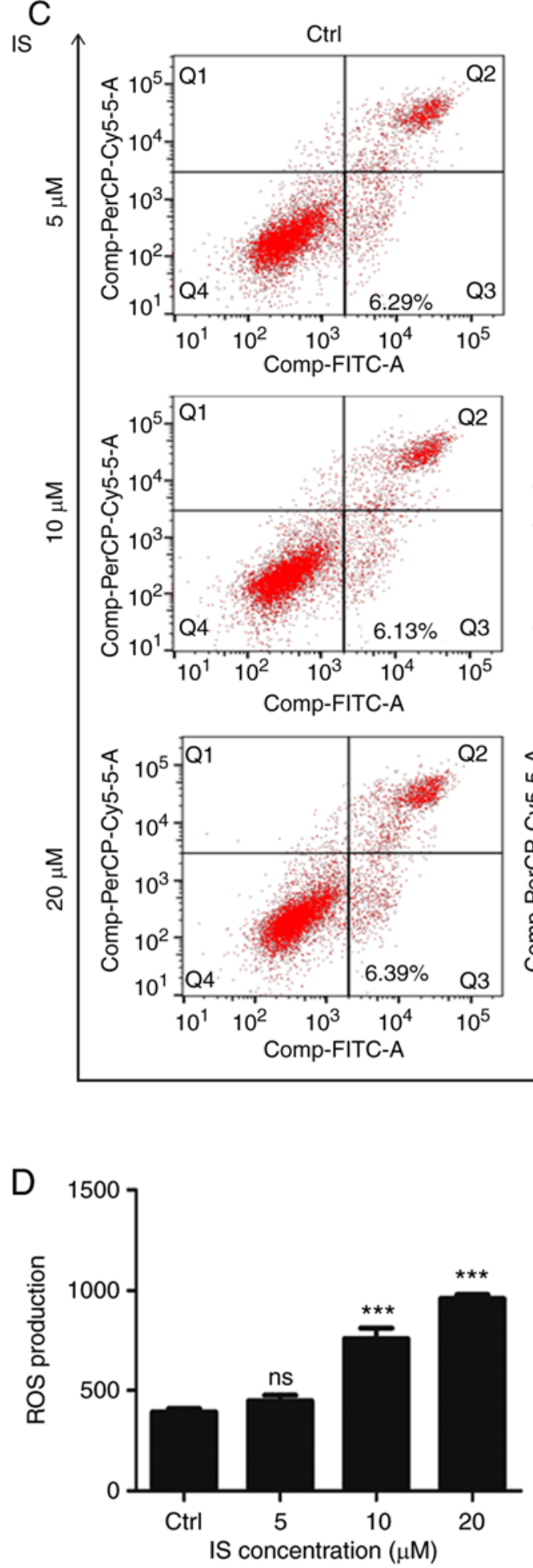
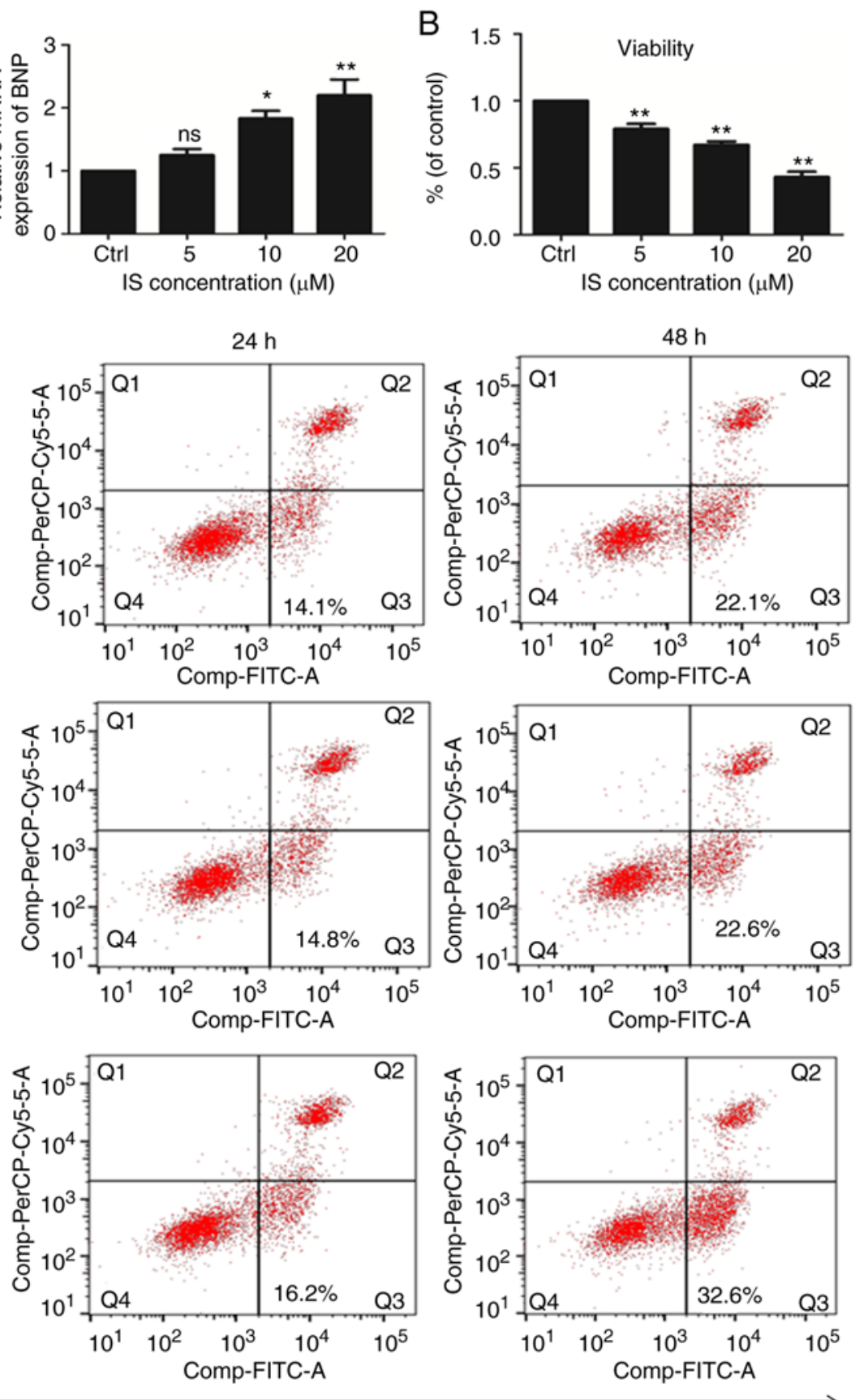

Time

E

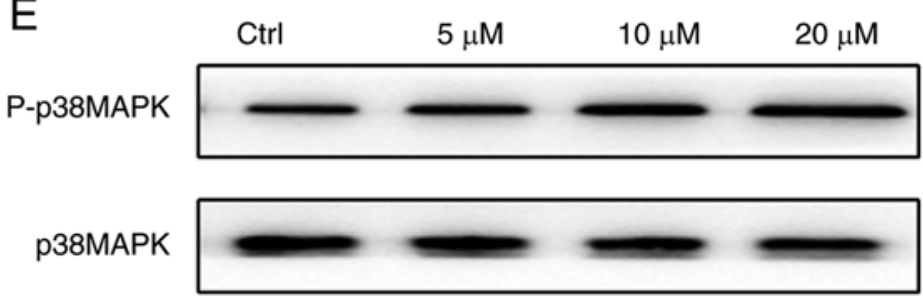

GAPDH

H9c2

Figure 6. Klotho downregulation activated the ROS/p38 MAPK signaling pathway. (A) mRNA levels of ANP and BNP in H9c2 cells after IS treatment. (B) Viability of H9c2 cells after IS treatment determined using Cell Counting Kit-8 assay. (C) H9c2 cells were treated with IS for 24 and $48 \mathrm{~h}$ and the results were analyzed using a flow cytometer. (D) ROS production in H9c2 cells after IS treatment. (E) Protein expression levels of p38 MAPK in H9c2 cells after IS treatment. 

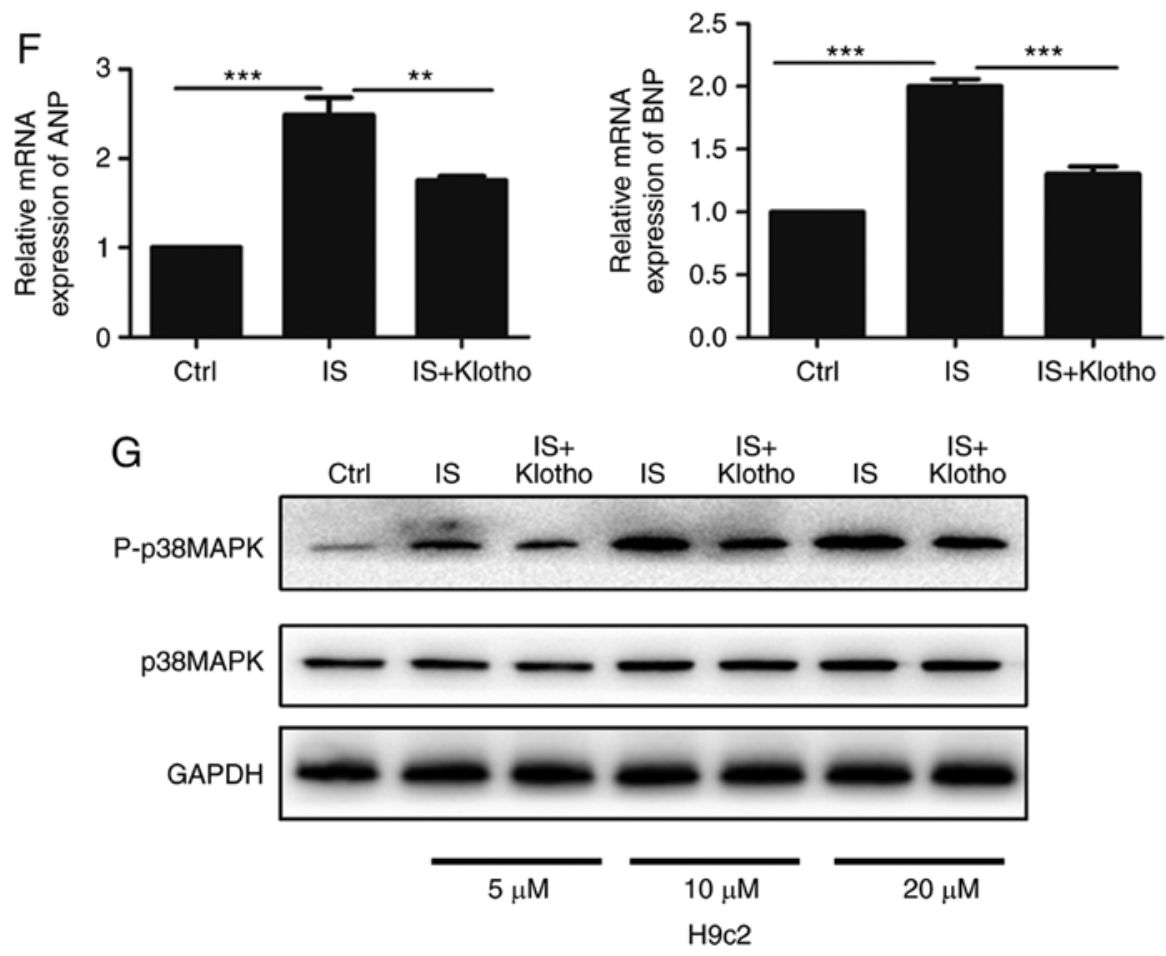

Figure 6 (Continued). Klotho downregulation activated the ROS/p38 MAPK signaling pathway. (F) mRNA levels of ANP and BNP in H9c2 cells after Klotho treatment detected using quantitative PCR. (G) Protein expression levels of p38 MAPK in H9c2 cells following Klotho treatment. ${ }^{*} \mathrm{P}<0.05$, ${ }^{* *} \mathrm{P}<0.01$ and ${ }^{* * *} \mathrm{P}<0.001$. ROS, reactive oxygen species; MAPK, mitogen-activated protein kinase; ANP, atrial natriuretic peptide; BNP, brain natriuretic peptide; IS, indoxyl sulfate.

uremic toxin that is correlated with sepsis (13). AST-120 (8\%) was administered to LPS-induced sepsis mice in order to remove the excess IS in serum. Notably, western blot analysis demonstrated that the expression of Klotho was improved in kidney and heart tissues (Fig. 5A and B). As shown by ELISA, the serum level of Klotho protein was also increased (Fig. 5C). IS injection was then administered to mice in order to simulate a similar septic model and it was found that Klotho protein was decreased in kidney and heart tissues (Fig. 5D and E). The ELISA data demonstrated that the serum level of Klotho protein was also decreased after IS injection (Fig. 5F). These results indicated that IS decreased the expression of Klotho protein in LPS-induced sepsis.

Klotho downregulation by IS activates the ROS/p38 mitogen-activated protein kinase (MAPK) signaling pathway in LPS-induced sepsis. To investigate the downstream effectors of Klotho in the myocardial damage of CRS in sepsis, H9c2 cardiomyocyte cells were treated with different IS concentrations to imitate Klotho downregulation. As shown in Fig. 6A, the expression levels of ANP and BNP were upregulated in the IS group. Fig. 6B shows that cell proliferation was decreased after IS treatment. In addition, the proportion of apoptotic cells was increased in the IS group (Fig. 6C). The production of ROS was also increased (Fig. 6D). As the ROS/MAPK signaling pathway plays an important role in CRS $(14,15)$, the level of MAPK was then detected. As shown in Fig. 6E, the phosphorylated p38 MAPK level was increased in IS group. Exogenous Klotho was added to the IS-treated H9c2 cells and it was found that the expression of ANP and BNP had a certain degree of reversal compared with the IS group (Fig. 6F). The expression of p38 MAPK was also decreased after Klotho treatment (Fig. 6G). These results indicated that decreased Klotho expression may cause the activation of the ROS/p38 MAPK signaling pathway, leading to myocardial damage.

\section{Discussion}

Myocardial injury caused by CRS is a common complication in sepsis patients and has a high mortality rate. Recent studies have indicated that myocardial depression in sepsis is caused by a number of factors (16). For example, indocyanine green-001 can attenuate endotoxemia-induced cardiac depression through the downregulation of the Wnt/ $\beta$-catenin signaling pathway (17). In addition, it has been reported that microRNA(miR)-135a is upregulated in sepsis-induced myocardial depression (18) and a further study confirmed that miR-135a aggravated sepsis-induced myocardial dysfunction by regulating the $\mathrm{p} 38 \mathrm{MAPK} /$ nuclear factor $-\kappa \mathrm{B}$ signaling pathway (18). In addition, high serum levels of soluble triggering receptor expressed on myeloid cells-1 have been confirmed to be negatively correlated with left ventricular ejection fraction in septic patients (19). However, the molecular mechanism of this myocardial injury has yet to be fully elucidated. The results of the present study confirmed that Klotho protein is a key molecule in myocardial disorders in sepsis patients. Klotho is a protein that is correlated with the suppression of several aging phenotypes (20-22). It has been reported that Klotho can affect phosphate and glucose metabolism, and has antioxidant, adipogenic and other actions (23). In addition, previous studies have demonstrated that Klotho protein has protective activity against cardiovascular disease 
and heart injury $(9,24)$. A study confirmed that adults with higher plasma Klotho have a lower risk of developing coronary artery disease (25). In addition, Klotho protein can inhibit canonical transient receptor potential 6 channels to reject pathological heart alterations (26). However, whether Klotho has a protective function against the myocardial damage of CRS in sepsis has yet to be elucidated. The present study found that Klotho protein was downregulated in the heart and kidney tissues of septic mice and that the serum level of Klotho was also significantly decreased. In addition, the myocardial function of LPS-induced septic mice was significantly improved after treatment with Klotho protein. These results suggested that Klotho played an important role in sepsis-associated myocardial injury. Therefore, this raises the question of what role the downregulation of Klotho protein expression plays in sepsis-associated myocardial injury. The data from the present study demonstrated that IS negatively regulated the expression of Klotho. It has been reported that IS can induce cell apoptosis through oxidative stress and the MAPK signaling pathway (27). In addition, a previous study confirmed that the protective function of Klotho protein in cardiovascular disease mainly depends on its antioxidative and antiapoptotic activity (9). The data from the present study confirmed that decreased Klotho induced the activation of the ROS/p38 MAPK signaling pathway, leading to cardiomyocyte apoptosis and finally resulting in myocardial damage.

\section{Acknowledgements}

Not applicable.

\section{Funding}

The present study was supported by Zhejiang Nature Science Fund (grant no.LY16H150005), the Medical Scientific Research Foundation of Zhejiang Province (grant no. WKJ-ZJ-1401) and Zhejiang Provincial Program for the Cultivation of High-level Innovative Health talents.

\section{Availability of data and materials}

All data generated or analyzed during this study are included in this published article.

\section{Authors' contributions}

FY initiated and designed the present study. FY and YF performed the experiments and interpreted the results. FY, YF and JY conducted all of the experiments. JC designed the experiments and revised the manuscript. All authors read and approved the final manuscript and agree to be accountable for all aspects of the research in ensuring that the accuracy or integrity of any part of the work are appropriately investigated and resolved.

\section{Ethics approval and consent to participate}

All experiments involving animals were approved by the Animal Care and Welfare Committee of Zhejiang Hospital.

\section{Patient consent for publication}

Not applicable.

\section{Competing interests}

The authors declare that they have no competing interests.

\section{References}

1. Krivan S, Kapelouzou A, Vagios S, Tsilimigras D, Katsimpoulas M, Moris D, Aravanis CV, Demesticha TD, Schizas D, Mavroidis M, et al: Increased expression of Toll-like receptors 2, 3, 4 and $7 \mathrm{mRNA}$ in the kidney and intestine of a septic mouse model. Sci Rep 9: 4010, 2019.

2. Aird WC: The role of the endothelium in severe sepsis and multiple organ dysfunction syndrome. Blood 101: 3765-3777, 2003.

3. Virzi GM, Clementi A, Brocca A and Ronco C: Endotoxin effects on cardiac and renal functions and cardiorenal syndromes. Blood Purif 44: 314-326, 2017.

4. Kotecha A, Vallabhajosyula S, Coville HH and Kashani K: Cardiorenal syndrome in sepsis: A narrative review. J Crit Care 43: 122-127, 2018.

5. Virzi GM, Clementi A, Brocca A, de Cal M, Marcante S and Ronco C: Cardiorenal syndrome type 5 in sepsis: Role of endotoxin in cell death pathways and inflammation. Kidney Blood Press Res 41: 1008-1015, 2016.

6. Clementi A, Virzi GM, Brocca A and Ronco C: The role of endotoxin in the setting of cardiorenal syndrome type 5. Cardiorenal Med 7: 276-283, 2017.

7. Blanco J, Muriel-Bombín A, Sagredo V, Taboada F, Gandía F, Tamayo L, Collado J, García-Labattut A, Carriedo D, Valledor M, et al: Incidence, organ dysfunction and mortality in severe sepsis: A Spanish multicentre study. Critic care 12: R158, 2008.

8. Abolghasemi M, Yousefi T, Maniati M and Qujeq D: The interplay of Klotho with signaling pathway and microRNAs in cancers. J Cell Biochem 120: 14306-14317, 2019.

9. Olejnik A, Franczak A, Krzywonos-Zawadzka A, Kaluzna-Oleksy M and Bil-Lula I: The biological role of klotho protein in the development of cardiovascular diseases. Biomed Res Int 2018: 5171945, 2018.

10. Oh HJ, Oh H, Nam BY, You JS, Ryu DR, Kang SW and Chung YE: The protective effect of klotho against contrast-associated acute kidney injury via the anti-oxidative effect. Am J Physiol Renal Physiol 317: F881-F889, 2019.

11. Livak KJ and Schmittgen TD: Analysis of relative gene expression data using real-time quantitative PCR and the 2(-Delta Delta C(T)) method. Methods 25: 402-408, 2001.

12. Hu MC, Shi M, Gillings N, Flores B, Takahashi M, Kuro-O M and Moe OW: Recombinant $\alpha$-Klotho may be prophylactic and therapeutic for acute to chronic kidney disease progression and uremic cardiomyopathy. Kidney Int 91: 1104-1114, 2017.

13. Veldeman L, Vanmassenhove J, Van Biesen W, Massy ZA, Liabeuf S, Glorieux $G$ and Vanholder R: Evolution of protein-bound uremic toxins indoxyl sulphate and p-cresyl sulphate in acute kidney injury. Int Urol Nephrol 51: 293-302, 2019.

14. Yu D, Li M, Tian Y, Liu J and Shang J: Luteolin inhibits ROS-activated MAPK pathway in myocardial ischemia/reperfusion injury. Life Sci 122: 15-25, 2015.

15. Lu S, Zhang Y, Zhong S, Gao F, Chen Y, Li W, Zheng F and Shi G: N-n-butyl haloperidol iodide protects against hypoxia/ reoxygenation injury in cardiac microvascular endothelial cells by regulating the ROS/MAPK/Egr-1 pathway. Front Pharmacol 7: 520, 2016.

16. Zanotti-Cavazzoni SL and Hollenberg SM: Cardiac dysfunction in severe sepsis and septic shock. Curr Opin Crit Care 15: 392-397, 2009.

17. Yousif NG, Hadi NR and Hassan AM: Indocyanine green-001 (ICG-001) attenuates Wnt/ $\beta$-catenin-induces myocardial injury following sepsis. J Pharmacol Pharmacother 8: 14-20, 2017.

18. Zheng G, Pan M, Jin W, Jin G and Huang Y: MicroRNA-135a is up-regulated and aggravates myocardial depression in sepsis via regulating $\mathrm{p} 38 \mathrm{MAPK} / \mathrm{NF}-\kappa \mathrm{B}$ pathway. Int Immunopharmacol 45: 6-12, 2017. 
19. Li Z, Zhang E, Hu Y, Liu Y and Chen B: High Serum sTREM-1 correlates with myocardial dysfunction and predicts prognosis in septic patients. Am J Med Sci 351: 555-562, 2016.

20. Kuro-o M, Matsumura Y, Aizawa H, Kawaguchi H, Suga T, Utsugi T, Ohyama Y, Kurabayashi M, Kaname T, Kume E, et al: Mutation of the mouse klotho gene leads to a syndrome resembling ageing. Nature 390: 45-51, 1997.

21. Reish NJ, Maltare A, McKeown AS, Laszczyk AM, Kraft TW, Gross AK and King GD: The age-regulating protein klotho is vital to sustain retinal function. Invest Ophthalmol Vis Sci 54: 6675-6685, 2013.

22. Xu Y and Sun Z: Molecular basis of klotho: From gene to function in aging. Endocr Rev 36: 174-193, 2015.

23. Flotyńska J, Uruska A, Araszkiewicz A and ZozulińskaZiółkiewicz D: Klotho protein function among patients with type 1 diabetes. Endokrynol Pol 69: 696-704, 2018.

24. Guo Y, Zhuang X, Huang Z, Zou J, Yang D, Hu X, Du Z, Wang L and Liao X: Klotho protects the heart from hyperglycemia-induced injury by inactivating ROS and NF- $\mathrm{BB}$-mediated inflammation both in vitro and in vivo. Biochim Biophys Acta Mol Basis Dis 1864: 238-251, 2018.
25. Semba RD, Cappola AR, Sun K, Bandinelli S, Dalal M, Crasto C, Guralnik JM and Ferrucci L: Plasma klotho and cardiovascular disease in adults. J Am Geriatrics Soc 59: 1596-1601, 2011.

26. Xie J, Cha SK, An SW, Kuro OM, Birnbaumer L and Huang CL: Cardioprotection by Klotho through downregulation of TRPC6 channels in the mouse heart. Nat Commun 3: 1238, 2012.

27. Lin YT, Wu PH, Tsai YC, Hsu YL, Wang HY, Kuo MC, Kuo PL and Hwang SJ: Indoxyl sulfate induces apoptosis through oxidative stress and mitogen-activated protein kinase signaling pathway inhibition in human astrocytes. J Clin Med 8: pii: E191, 2019.

This work is licensed under a Creative Commons Attribution-NonCommercial-NoDerivatives 4.0 International (CC BY-NC-ND 4.0) License. 